\title{
Controversies and Provocative Statements
}

- The Arecibo ALFALFA survey won't find a different source population from HIPASS because large telescopes are not suited to blind HI surveys. Disney.

- Yes they will. Giovanelli, Haynes, Kent.

- Blind surveys find that all HI galaxies have the same HI Column density. And it's not an instrumental or selection effect. Disney.

- Yes it is. Its because of your instrumentation. Giovanelli from the floor.

- Many claimed HI optical identifications could be wrong due to strong clustering. Disney

- HI may be missing because of spin temperature freezeout. Disney

- There's no feezeout. Taylor and Webster have shown that all HI clouds of galaxy mass should form stars. Taylor - floor.

- My simulations show there is plenty of room for Dark Galaxies to form. Davies.

- VIRGOHI21 is a good Dark Galaxy candidate. Davies.

- No it isn't. It's tidal debris. Haynes, Giovanelli, Duc, Tully.

- Whatever it is it isn't tidal debris. Disney.

- Davies' simulations of VIRGOHI21 do not match our deep Arecibo data now in press. Giovanelli.

- My simulations of tidal encounters can explain VIRGOHI21 without a Dark Galaxy. Duc

- No they don't fit in detail. They only fit the Arecibo data which doesn't have enough resolution. Minchin (showed fit).

- Resolution doesn't matter. Haynes from floor.

- Chandra picks up Oxygen lines of hot X-ray gas consistent with the predicted Warm Hot Intergalactic Medium. Mathur

- But they're not detected by XMM. Audience.

- It's a problem with the XMM gratings. Mathur

- All objects more than about 120 pc in size appear to have Dark Matter halos of more than $4 \times 10^{7} \mathrm{M}_{\odot}$. Wyse.

- Spirals must be shaped by several physical processes. Salucci.

- ALFALFA will find hundreds of HI clouds with masses less than $10^{7} \mathrm{M}_{\odot}$ and this will yield an accurate HI mass function. Haynes.

- No it won't. Anyway you would need tens of thousands, as with the optical galaxy LF. Disney

- What is a Dark Galaxy? I define it as a stable disc. There aren't any in HIPASS. Taylor.

- The baryonic Tully Fisher relation fits down to $10 \mathrm{~km} / \mathrm{sec}$. with a slope of 0.25 and does not fit the CDM simulations of Mo et al. which predict 0.33. McGaugh.

- All the baryons in galaxies are accounted for. McGaugh

- The Local Void is really empty. Tully.

- Simulations are not data. Impey

- There is no compelling evidence for missing baryons if you take diffuse light into account, Gonzalez. 
- Lensing suggests for E/SO galaxies that there must be a conspiracy between halos and light. Koopmans.

- High resolution simulations of galaxy formation in groups strongly suggests that many of the minihalos predicted by CDM ought to be visible. Nelson

- "Catching black cats in a dark room is a very enthusiastic occupation". Karachentsev

- The Toomre Criterion is not valid in galaxies. Schaye

- Because of the UV background halos below $7 \times 10^{9} \mathrm{M}_{\odot}$ will be deficient in baryons. Hoeft

- dSphs must be continuously stripped, Grebel.

- There is no evidence of quenching in the Local Group Dwarfs. Grebel.

- The published dSph. masses could well be heavily contaminated by stripped and foreground stars and the true $\mathrm{M} / \mathrm{L}$ ratios could be low. Klimentowski.

- With the discovery of ever more dim and dwarf galaxies we are only a factor 4 short of the CDM simulations. Geha.

- The Fornax cluster has a flat faint end LF, contrary to earlier work. Mieske.

\section{IAU Colloquium 171 'The Low Surface Brightness Universe', Cardiff, 1998 Controversies and Provocative Statements}

- It's quite safe to walk on the beach. Disney

- LSBGs give rise to none/all of QSO absorption lines. Lanzetta/Linder

- Many of you are doing your visibility corrections wrong. Disney

- Why did we not find red LSBGs before. O'Neil

- Is $(M / L)_{L S B}=(M / L)_{H S B}$ ?

- Surface brightness distribution is bi-modal. Tully

- HSB is a temporary phenomenon. Bothun

- Do LSBGs and HSBGs live in the same halo's? de Blok

- Will the inter-galactic radiation field kill low $N_{H I}$ ? Briggs

- Does Dark Matter exist? McGaugh

- Massive LSBGs $\left[M_{B}<-20\right]$ are exotic. Tully

- Multi-beams will not find invisible galaxies. Tully

- (g)Es are caused by cannibalism. Richter

- Do all disks follow the same T-F relation?

- We would not see Malin 1 at the distance of M31.

- Morphological separation of Sd/Sn/Ic is meaningless. McGaugh

- Why did Bothun et al. find Malin 1?

- How can there be large HI discs mostly devoid of stars, but no such discs with no stars?

- $\mu(R)$ is meaningless for dwarfs. Brosch

- Are blue LSBGs young?

- Supernovae WILL NOT clean gas out of dwarfs.

- How do stars form if Jeans length greater than scale length? Bothun

- $\alpha=-1.5$ is simply a consequence of selection effects. Disney

- Most of the galaxies in our neighbourhood are undiscovered. Disney

- All the members of the Local Group have been discovered. Irwin, Kraan-Korteweg 
- LSBGs are low angular momentum systems.

- There are very few completely dark HI clouds. Zwaan, Tully

- Steep $\alpha$ 's in clusters are due to background contamination. Huchra

- HDF shows no evidence of a large population of LSBGs. Ferguson

- $z=0.1$ is the best place to look for LSBGs. Dalcanton

- dEs and dIs are more extended in clusters than 'ordinary' galaxies. Davies, Phillipps

- Red LSBGs are younger than blue LSBGs. Bell

- Maximum disc models do not work for LSBGs. de Blok

- How do stars form at all well below Kennicutt column density threshold? van Zee, Bothun

- Bars in LSBGs are common; how did they form? Knezek

- Shredded LSBGs are not progenitors of BCD. Salzer

- Super thin galaxies are LSBGs seen edge-on. van Driel

- Weak MgII absorbers are due to LSBGs. le Brun

- Optical Cirrus can look like a LSBG; we have to be careful. Guhathakurta

- UV is the best place to look for diffuse light from space. Henry

- There exists galaxies at $z=6.8$. Lanzetta

- But $(1+z)^{4}$ is 9 magnitudes; they should be invisible. Disney

- HDF fluctuations do not allow many LSBGs. Vogeley

- How do we get absorption spectra of very LSBGs?

- The 'flat' $\varphi\left(\mu_{0}\right)$ of McGaugh is NOT the $\varphi\left(\mu_{0}\right)$ distribution at a given $h$ or $L$ nor integrated over all galaxies. de Jong

- Does CDM make ANY predictions that cannot be fudged? 